\title{
BMJ Open Evaluation of a decision aid for incidental genomic results, the Genomics ADvISER: protocol for a mixed methods randomised controlled trial
}

Salma Shickh, ${ }^{1,2}$ Marc Clausen, ${ }^{2}$ Chloe Mighton, ${ }^{1,2}$ Selina Casalino, ${ }^{2,3}$ Esha Joshi, ${ }^{1,2}$ Emily Glogowski, ${ }^{4}$ Kasmintan A Schrader, ${ }^{5}$ Adena Scheer, ${ }^{2}$ Christine Elser, ${ }^{6}$ Seema Panchal, ${ }^{7}$ Andrea Eisen, ${ }^{8}$ Tracy Graham, ${ }^{8}$ Melyssa Aronson, ${ }^{7}$ Kara M Semotiuk, ${ }^{7}$ Laura Winter-Paquette, ${ }^{7}$ Michael Evans, ${ }^{1,2}$ Jordan Lerner-Ellis, ${ }^{1,7}$ June C Carroll, ${ }^{1,7}$ Jada G Hamilton, ${ }^{9}$ Kenneth Offit, ${ }^{9}$ Mark Robson, ${ }^{9}$ Kevin E Thorpe, ${ }^{10,11}$ Andreas Laupacis, ${ }^{1,2}$ Yvonne Bombard ${ }^{1,2}$

To cite: Shickh S, Clausen M, Mighton C, et al. Evaluation of a decision aid for incidental genomic results, the Genomics ADvISER: protocol for a mixed methods randomised controlled trial. BMJ Open 2018;8:e021876. doi:10.1136/ bmjopen-2018-021876

- Prepublication history and additional material for this paper are available online. To view these files, please visit the journal online (http://dx.doi. org/10.1136/bmjopen-2018021876).

Received 24 January 2018 Revised 18 February 2018 Accepted 20 February 2018

Check for updates

For numbered affiliations see end of article.

Correspondence to Dr Yvonne Bombard; yvonne.bombard@utoronto.ca

\section{ABSTRACT}

Introduction Genome sequencing, a novel genetic diagnostic technology that analyses the billions of

base pairs of DNA, promises to optimise healthcare through personalised diagnosis and treatment. However, implementation of genome sequencing faces challenges including the lack of consensus on disclosure of incidental results, gene changes unrelated to the disease under investigation, but of potential clinical significance to the patient and their provider. Current recommendations encourage clinicians to return medically actionable incidental results and stress the importance of education and informed consent. Given the shortage of genetics professionals and genomics expertise among healthcare providers, decision aids (DAs) can help fill a critical gap in the clinical delivery of genome sequencing. We aim to assess the effectiveness of an interactive DA developed for selection of incidental results.

Methods and analysis We will compare the DA in combination with a brief Q\&A session with a genetic counsellor to genetic counselling alone in a mixedmethods randomised controlled trial. Patients who received negative standard cancer genetic results for their personal and family history of cancer and are thus eligible for sequencing will be recruited from cancer genetics clinics in Toronto. Our primary outcome is decisional conflict. Secondary outcomes are knowledge, satisfaction, preparation for decision-making, anxiety and length of session with the genetic counsellor. A subset of participants will complete a qualitative interview about preferences for incidental results.

Ethics and dissemination This study has been approved by research ethics boards of St. Michael's Hospital, Mount Sinai Hospital and Sunnybrook Health Sciences Centre. This research poses no significant risk to participants. This study evaluates the effectiveness of a novel patient-centred tool to support clinical delivery of incidental results. Results will be shared through national and international conferences, and at a stakeholder
Strengths and limitations of this study

The study is hypothetical and predicted behaviour may differ from actual behaviour

- This study will assess the effectiveness of a novel patient-centred tool to support clinical delivery of incidental sequencing results, filling a significant gap in light of limited genomics expertise and resources.

- Our study will include a qualitative component that will allow us to gain insight into participants' selection of incidental findings. The results of this could help guide clinicians in counselling patients to facilitate shared-decision making.

workshop to develop a consensus statement to optimise implementation of the DA in practice.

Trial registration number NCT03244202; Pre-results.

\section{INTRODUCTION}

Genome sequencing, a test designed to analyse the billions of base pairs of DNA in the human genome, can improve diagnosis and inform targeted treatments, cornerstones of personalised healthcare. ${ }^{1-3}$ The 100000 Genomes Project, which aims to sequence the genomes of 100000 National Health Services patients, aims to increase the utilisation of genome sequencing in mainstream healthcare. ${ }^{4}$ However, widespread implementation of genome sequencing faces challenges, including the lack of a consensus on how to counsel patients on the wide range of incidental results generated.

Incidental sequencing results are genetic mutations that are unrelated to the disease 
under investigation but of potential clinical significance to the patient and their healthcare provider. The return of incidental results has numerous practical and ethical implications, especially with regard to which types of results to return to patients. This has generated significant controversy and there continues to be variation in the practice of returning incidental findings across laboratories and clinics. $^{56}$ The American College of Medical Genetics and Genomics (ACMG) recommends that, at minimum, patients undergoing genome sequencing be offered the option to receive results for 59 medically actionable genes which the ACMG defined as those associated with a high penetrance (high likelihood of disease developing) and for which there is available medical intervention. ${ }^{7}$ An example of a gene in the ACMG list is KCNQ1, which is associated with long QT syndrome, a condition that can result in an arrhythmia, syncope, cardiac arrest and sudden death. Preventive measures available for individuals identified to have long QT syndrome include beta-blockers, cardiac sympathetic denervation surgery or an implantable cardioverter defibrillator with the goal to prevent syncope, cardiac arrest and sudden death. ${ }^{8}$ The Clinical Genome Resource (ClinGen), a central resource on the clinical relevance of genes and variants, is curating a database which now contains over 100 genes that they deem as having some level of clinical actionability. ${ }^{6}$ Furthermore, data from public preference studies on the return of incidental results have consistently demonstrated that individuals prefer to receive incidental results beyond those that are classified by professionals and researchers as medically actionable. ${ }^{9}$

The volume of incidental results is substantial. Studies have shown that $96 \%$ of participants undergoing genome sequencing are found to have pharmacogenetics results, variants related to drug side effects. ${ }^{10}$ Additionally, $84 \%-100 \%$ are found to have carrier results, which inform patients that although they are unaffected with the disease, they are at risk to have an affected child if their partner is also a carrier of the same disease. ${ }^{3} 10$ Furthermore, some diseases identified through genome sequencing will have available prevention strategies or treatments, whereas others will not. Therefore, it could be argued that patients require extensive genetic counselling (GC) for the myriad of possible incidental genomic results, the implications for their health as well as the health of their family members, in order to make an informed decision.

As the use of genome sequencing increases, the demand for GC will increase. Traditional GC methods require hours of education and counselling, costly and infeasible given the limited numbers of genetic counsellors. ${ }^{11}{ }^{12}$ Novel strategies to educate patients and reduce the burden on genetic counsellors to support patients' pretest preferences for incidental results are needed. Decision aids (DAs) may reduce the amount of time genetic counsellors spend with patients in pretest counselling sessions and guide patients in the selection of incidental results that they wish to receive before they undergo genome sequencing.
DAs in GC improve pretest patient education. They can assist in educating patients about genetic testing while provoking thoughts around the benefits and limitations of testing. ${ }^{13-18}$ DAs are effective in increasing knowledge in patients undergoing prenatal and cancer testing, ${ }^{14} 15$ and in reducing cancer-related distress in women with a BRCA1 or BRCA2 gene mutation. ${ }^{16}$ Interactive online DAs result in equal or improved knowledge acquisition over conventional counselling. ${ }^{17}$ Yet there are no DAs to support patient selection of incidental results. Other decision support tools available review genome sequencing prior to testing ${ }^{18}$ but do not focus on incidental results.

We created an interactive DA called the 'Genomics ADvISER' (a Genomics decision AiD about Incidental SEquencing Results) (figure 1) to guide patients' selection of incidental results (www.genomicsadviser.com) based on the evidence-based Ottawa Decision Support Framework (ODSF) $)^{19-22}$ and guidelines for returning incidental results described elsewhere. ${ }^{23}$ Briefly, the DA consists of a background whiteboard video that reviews key concepts of genome sequencing and risks and benefits of learning incidental sequencing results. This is followed by preparation for decision-making using a values clarification exercise (with feedback of their preferences) and a knowledge quiz to reinforce the key concepts. The DA ends by asking participants to select categories they want to learn from among five categories of incidental results, consistent with conceptual 'binning' guidelines ${ }^{24-26}$ (see figure 2). The DA was evaluated in a usability study in which the DA demonstrated strong face validity, acceptability and high content comprehension. All usability study participants felt that the DA had enough information to make a decision and they would recommend the DA to others. ${ }^{26}$

\section{Study aim}

The primary aim of the study is to evaluate the effectiveness of a DA developed for pretest GC to guide the selection of incidental sequencing results. We hypothesise that the DA in conjunction with a brief Q\&A session with a genetic counsellor will result in less decisional conflict (primary endpoint), less time spent with a genetic counsellor and improved patient experience (knowledge, satisfaction, preparedness, shared decision-making) and health outcomes (reduced anxiety) compared with patients undergoing GC alone. The secondary aim of the study is to explore the factors affecting the selection of incidental findings through qualitative interviews.

\section{METHODS AND ANALYSIS \\ Study design}

This is a mixed-methods randomised controlled trial (RCT) that will evaluate whether an online DA followed by a brief Q\&A session with a genetic counsellor reduces decisional conflict compared with GC alone. Participants will view the DA and have a brief Q\&A session with a genetic counsellor or GC only, and be asked to select 


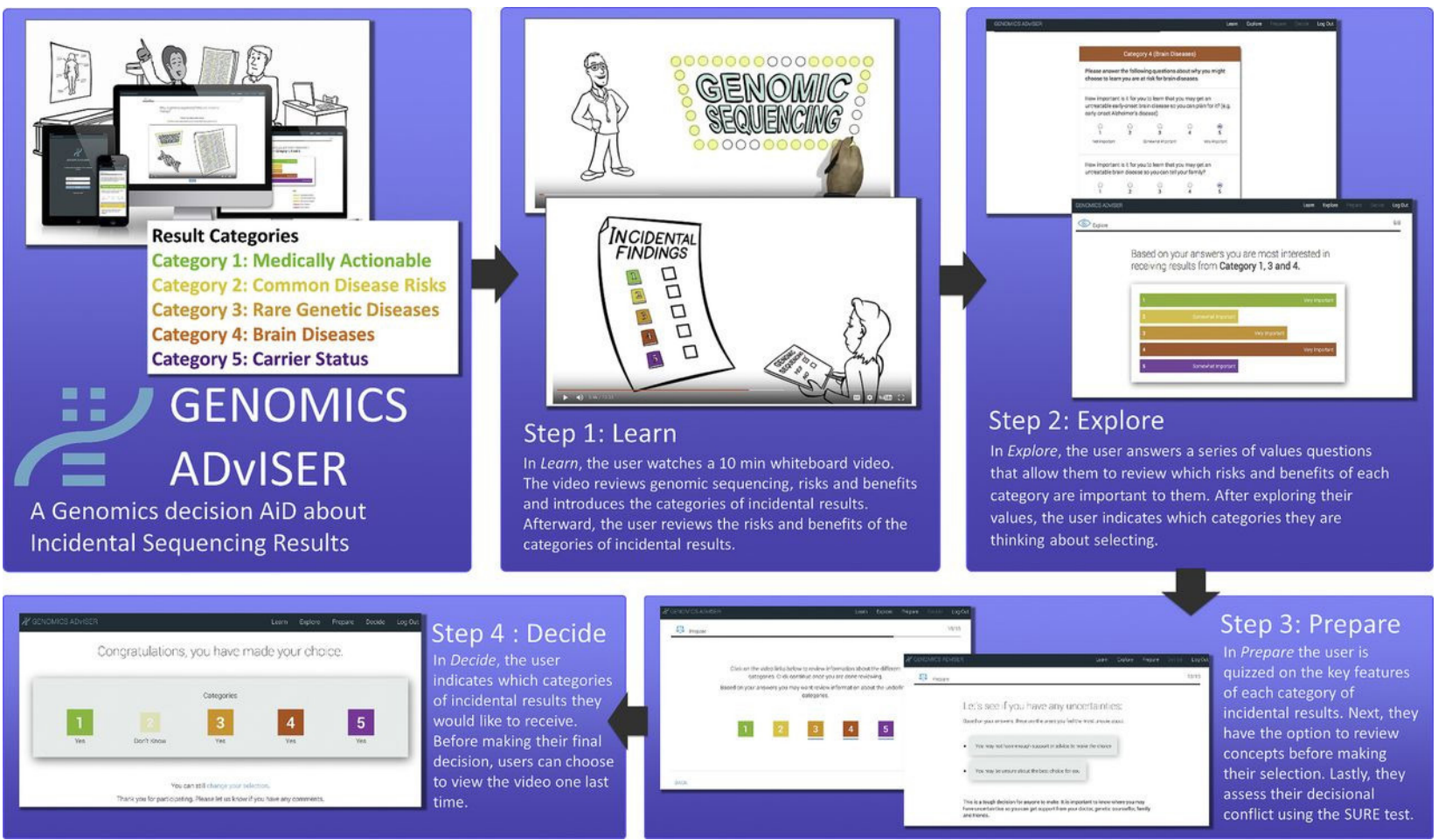

Figure 1 The Genomics ADvISER.

incidental results that they would like to learn if they were offered genome sequencing and incidental results. The goal of the study is to evaluate the effectiveness of the DA in pretest counselling so participants will not undergo genome sequencing or be provided with any results; therefore, the study is hypothetical. However, participants will learn about genome sequencing, which may be beneficial to them since they may be eligible for genome sequencing at some point in the future. A subset of participants will complete a follow-up qualitative interview.

\section{Study setting}

The main study site is St. Michael's Hospital in Toronto, Ontario. Patients will be recruited from cancer genetics clinics at Mount Sinai Hospital and Sunnybrook Health Sciences Centre located in Toronto, Ontario. The

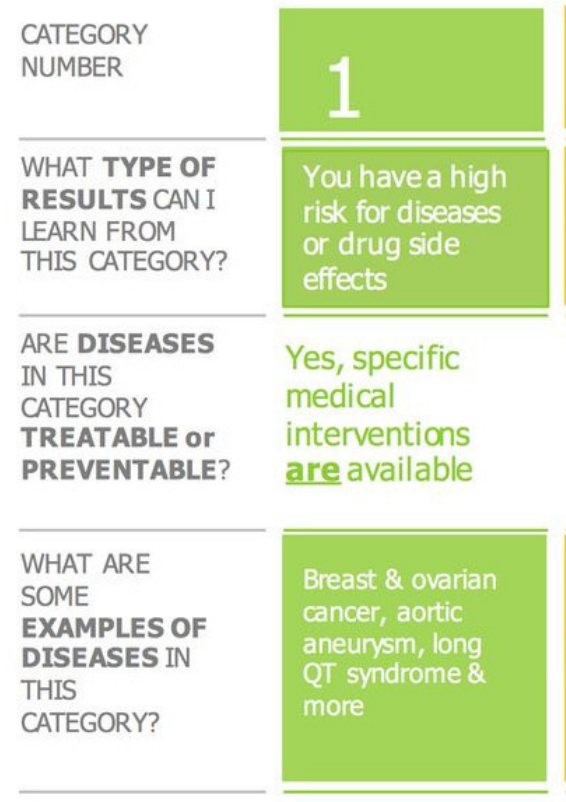

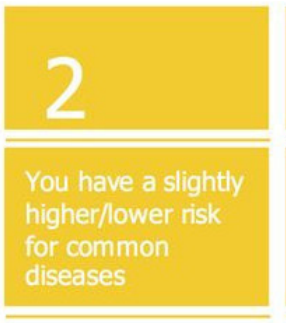

No, specific medical interventions are not available for diseases in these categories

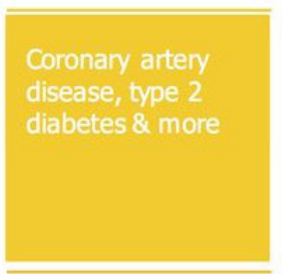

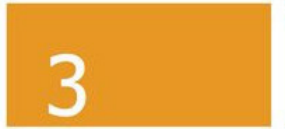

You already have diseases, or have a higher risk for some

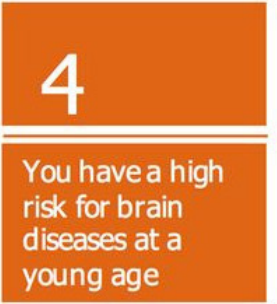

Birth defects (in kidneys, bones), Degenerative diseases (nerve damage, blindness) \& more
Early-onset

Alzheimer's, earlyonset Parkinson's, late-onset Krabbe disease

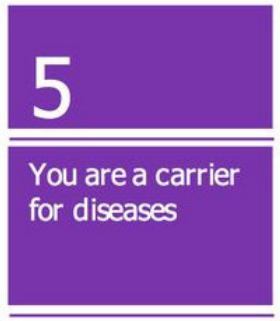

Medical interventions are availablefor some diseases

Carrier for cystic fibrosis, sickle

cell disease, phenylketonuria \& more

Figure 2 Binning tool. 
recruitment period started in September 2016 and is expected to end by March 2018.

\section{Participants}

Eligibility criteria were developed to reflect future clinical eligibility for genome sequencing; participants will be patients whose personal and family history is suggestive of an inherited cancer syndrome but firsttier genetic testing for classic causative mutations (eg, $B R C A 1 / 2)$ has been negative. Genome sequencing may be used as a second-tier test in identifying the causative mutation. Patients must be 18 years of age or older, be able to speak and read English, have internet access and be internet literate. Patients will be excluded if they have recurrent metastatic cancer (stage 4), since studies indicate that incidental results are perceived as burdensome to this population given their ongoing health challenges. ${ }^{27}$ Patients and their family members who participated in the usability study of the DA that preceded this RCT will also be excluded, to reduce confounding. ${ }^{23}$

\section{Recruitment}

Eligible patients will be informed about the study by a genetic counsellor during their clinic visit or over the phone when their negative genetic test results are returned. During an in-person clinic visit, interested patients will be given a copy of the study invite package. Those who are interested will be asked to fill out the contact form that will be given to the study coordinator who will follow-up with the patient. If their negative results are disclosed over the phone, the genetic counsellor will complete the study contact information for interested patients. The study coordinator will contact interested patients on the phone and explain the study further. Patients will also have the option of contacting the study coordinator directly and will be given the study coordinator's phone and email contact information by the genetic counsellor.

\section{Procedure for qualitative recruitment}

All consented participants will be invited to participate in qualitative interviews. Participants will be able to refuse to take part in the qualitative component and still participate in the RCT. We will interview up to 40 participants in total across both arms of the study, a typical sample size for grounded theory studies and sufficient to reach thematic saturation. ${ }^{28-30}$ We will first purposively sample participants across the arms of the study, and then theoretically sample based on emerging findings. ${ }^{31} 32$

\section{Data collection}

After being consented by the study coordinator, participants will complete a baseline questionnaire including demographic information, cancer history, genome sequencing knowledge and anxiety. Medical history will be collected from participants' medical charts with their consent. The study coordinator will access medical chart information for each participant from the recruiting clinic which will be used to describe the study population and confirm cancer and genetic testing history. The study coordinator will provide the study genetic counsellors with the patient's medical history and genetic testing history prior to the GC sessions.

Following the consent procedure and baseline data collection, participants will be randomised into the control arm (GC) or intervention arm (DA+abrief Q\&A session with a genetic counsellor) of the study (figure 3).

Participants from each clinic will be randomised separately to ensure that we have an even distribution of patients represented from all clinics in both arms of the study. A biostatistician will create a computer-generated randomisation list in a 1:1 ratio with random permuted blocks of varying sizes. The biostatistician will put all of the allocations into envelopes and seal them. The list will be given to a research assistant who does not have any role in the recruitment or tracking of participants in the study. These envelopes will be kept in a locked cabinet in the study office and will be retrieved by the study coordinator

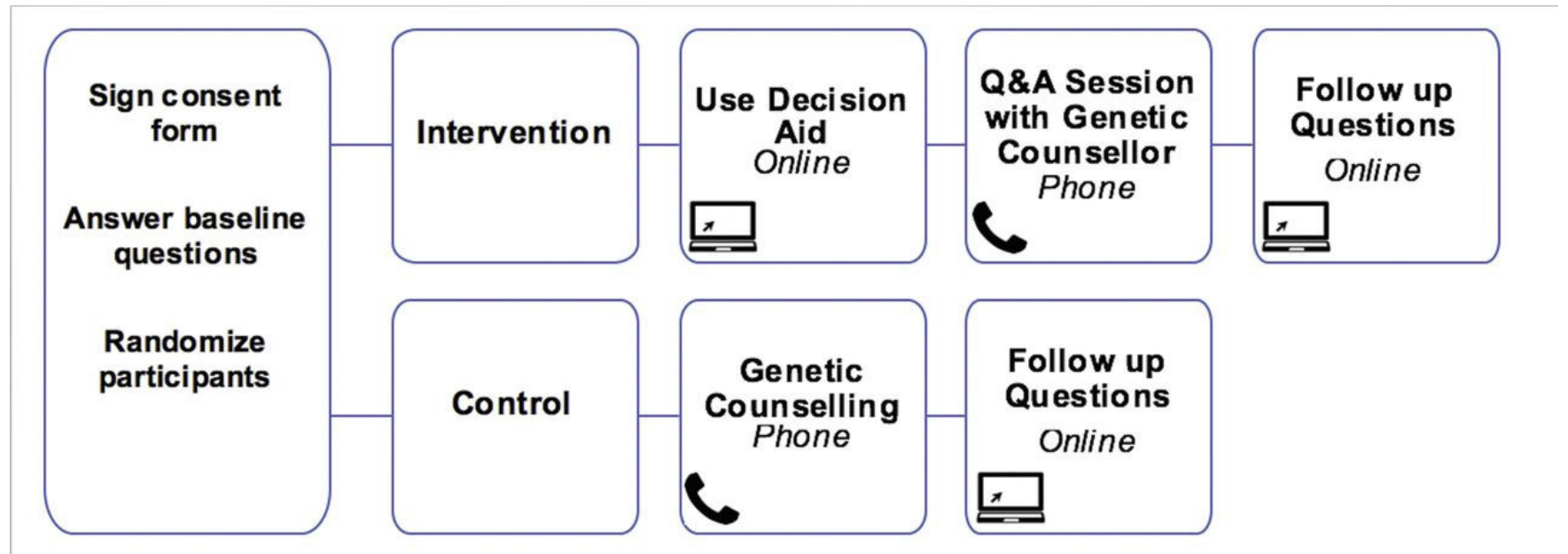

Figure 3 Study flow. 
when needed. They will only be opened after each patient is consented and has completed the baseline measures.

For the GC components of the study, six genetic counsellors (three for control and three for intervention) will be trained prior to the study on the scripts to ensure consistency in the content and delivery of the counselling materials.

With regard to measures, our primary outcome is decisional conflict, assessed via the validated Decisional Conflict Scale (DCS) ${ }^{33}$ consistent with the ODSF. ${ }^{22}$ Secondary outcomes include knowledge, satisfaction, anxiety and length of GC session. Knowledge will be measured by a genome sequencing questionnaire ${ }^{34}$ and a set of internally developed knowledge questions on incidental results. Satisfaction will be measured using the Satisfaction with Decision (SWD $)^{35}$ scale and the Preparation for Decision Making (PrepDM) scale. ${ }^{36}$ Anxiety will be measured using the state subscale of the State-Trait Anxiety Inventory (STAI), ${ }^{37}$ a commonly used psychological assessment tool to measure state anxiety in adult populations including those with chronic conditions. ${ }^{38}$ Both the control and intervention GC sessions will be recorded and the length of the sessions will be documented. This will allow us to assess if the DA reduces consultation time, which could help to inform resource needs in the clinical implementation of genome sequencing.

\section{Control arm}

After participants have been consented and randomised into the control arm, the study coordinator will schedule them for their phone GC session within 14 days. Prior to the phone GC session, the study coordinator will speak with the genetic counsellor over the phone and share relevant participant details. This would include participant's name, recruiting clinic, personal and family history of cancer as well as the type of genetic testing they have undergone. Details regarding their cancer history and genetic testing would be important for the counsellor to know if the participants have questions related to the genetic testing they have undergone.

After scheduling the GC session, the study coordinator will send an email to participants that includes a set of standard counselling visual aids (see online supplementary file 1) that will be used during the phone counselling session as well as the link and website login code where they will complete the online self-administered measures immediately after their session. The Genomic Counselling Guide is an eight-page booklet designed by the National Society of Genetic Counselors. ${ }^{39}$ The booklet includes visuals of cells, genes, chromosomes and a table summarising the five categories of incidental results that will be referred to during the GC session (figure 2). This is consistent with standard GC practice where visual aids are commonly used.

A script of genome sequencing topics will standardise each GC discussion covering educational content to enable patients to select categories of incidental sequencing results (see online supplementary file 2).
Participants in the control group will not view the DA video or complete the values clarification exercise or knowledge quiz. At the end of the GC session, the genetic counsellor will ask the participant to select which categories of incidental results they would like to receive if they were offered genome sequencing and record these on the data collection form. The data collection form includes notes taken by the genetic counsellor from the discussion with the study coordinator (participant study number, cancer history, genetic testing history) as well as the participants' category selections, notes about questions the participants asked during the counselling session and the start and end time of the session. The genetic counsellor will complete the form and submit it to the study coordinator for each participant. After the session, the genetic counsellor will ask the participants to complete the online self-administered measures (decisional conflict, genome sequencing knowledge, satisfaction with decision-making and anxiety).

\section{Intervention arm}

After participants have been consented and randomised into the intervention arm, the study coordinator will provide them with a date for their brief phone Q\&A session with a genetic counsellor within 14 days and send them an email with a link to the online DA along with a unique login code. This email will instruct the intervention participants to $\log$ on to the DA programme, view the DA and complete online self-administered measures 1 hour before their scheduled meeting with the genetic counsellor. The DA responses will be recorded in a database through an online provider. Prior to the brief phone Q\&A session, the study coordinator will speak with the genetic counsellor over the phone and share relevant participant details (such as the patient's cancer history).

After completing the online DA and measures, participants will speak with a genetic counsellor over the telephone. During the session, the genetic counsellor will use a standardised script (see online supplementary file 3) of questions and will have access to the participant's incidental result selections from the DA. At the end of the session, the genetic counsellor will ask the participants to complete another set of online self-administered measures (decisional conflict, genome sequencing knowledge, satisfaction with decision-making and anxiety). After the session with the participant, the genetic counsellor will complete the post session data collection form and submit it to the study coordinator.

\section{Qualitative component}

Once participants have completed the quantitative component, the study coordinator will follow-up with participants who indicated an interest in the qualitative component over the phone. The coordinator will confirm their interest, and will schedule the telephone interview if they elect to proceed. The coordinator will call the participant at the scheduled time for the interview and, using an interview guide, will explore factors that affected that 
participant's selection of incidental results (see online supplementary file 4 ). The interview guide will be developed based on a literature review and expert input, and modified based on data analysis. The interview guide will specifically explore: their interest in learning the categories they selected, factors that led them to select or not select each category and how they feel results from the categories they selected will be useful to them.

These interviews will be audio recorded and will take about 1 hour. The interviewer will take field notes immediately after each interview and interviews will also be transcribed verbatim.

\section{Data analysis and statistical methods Quantitative}

The analysis of outcomes will follow the intention-to-treat approach. Mean DCS, SWD, PrepDM and STAI scores and session length will be compared using a t-test. The study requires 64 patients/arm to detect the minimal clinically important difference of 0.3 using the DCS, assuming an SD of 0.6, an alpha of 0.05 (two-sided) and power of $0.8 .^{4041}$

Knowledge scores will be assessed by summing the number of correct responses to the questions, and compared using t-tests. Linear regression will be used in a secondary analysis to account for the following known predictors for decisional outcomes: age, gender, education and medical history (presence or absence of cancer). Secondary exploratory analyses will compare the mean DCS, knowledge, SWD, PrepDM and STAI scores before and after GC in the intervention arm to explore the additional benefit of speaking with a genetic counsellor after the DA. Un/adjusted mean differences and 95\% CI will be reported. We will use descriptive statistics to report participants' characteristics.

\section{Qualitative}

Qualitative data analysis will draw on grounded theory methodology. ${ }^{42}$ The qualitative data will be analysed as the study progresses and preliminary themes will be formed. Typical of constant comparison techniques, qualitative data will be analysed concurrent with data collection to explore preliminary themes and revise the interview guide. ${ }^{43-47}$ We will sort the data by searching for themes/patterns and variations within and across interviews using NVivo (V.10). Coding, which is the first stage in the analysis process, will involve 'labeling' the data with descriptive codes. Two team members will independently code each transcript. Consensus on coding will be reached through comparison and discussion among these members. Inter-rater agreement and kappa coefficients will be calculated to assess intercoder reliability. The second stage will involve constant comparison, where codes and their content will be compared across interviews to discern common and divergent themes and issues. ${ }^{43-46}$ The final stage is selective coding, which integrates all the codes under a central phenomenon to build a theory. Validation methods include triangulation and member checking. ${ }^{478}$

\section{ETHICS AND DISSEMINATION \\ Ethics}

This research poses no more than minimal risk to participants.

Informed consent will take place over the phone and will be recorded. All participants will receive a copy of the consent for their own records. The study coordinator will review the consent form in detail, and answer any questions regarding the study. Audio recordings of the phone consents will be kept in a locked file in the study office of St. Michael's Hospital. The study coordinator will sign the consent checklist, which will be kept in a separate location from other study materials, in the study offices in St. Michael's Hospital. The consent will ask for permission for access to medical records at recruiting clinics and permission to recontact participants for future studies. All information collected during this study, including personal information, will be kept confidential.

\section{Dissemination}

This research evaluates a novel patient-centred tool to support clinical delivery of incidental sequencing results, the Genomics ADvISER. The Genomics ADvISER will be used as the decision support tool among patients having genome sequencing to select incidental results, which will be returned to them as part of another RCT trial which will assess the preferences, outcomes and costs of returning incidental results. We will present the data from both trials at a stakeholder workshop to develop a consensus statement on the pathway to care in order to optimise implementation of the DA in practice. Stakeholders will include key opinion leaders/champions and end-users such as genetic counsellors, geneticists, oncologists, patients and clinical laboratories. Research articles based on the results will be written and submitted for publication in peer-reviewed journals. Additionally, results will be shared at national and international genetics and policy conferences.

\section{Author affiliations}

${ }^{1}$ University of Toronto, Toronto, Ontario, Canada

${ }^{2}$ St. Michael's Hospital, Toronto, Ontario, Canada

${ }^{3}$ Faculty of Science, McMaster University, Hamilton, Ontario, Canada

${ }^{4}$ GeneDx, Gaithersburg, Maryland, USA

${ }^{5}$ Hereditary Cancer Program, BC Cancer Agency, Vancouver, British Columbia, Canada

${ }^{6}$ University Health Network, Toronto, Ontario, Canada

${ }^{7}$ Mount Sinai Hospital, Sinai Health System, Toronto, Ontario, Canada

${ }^{8}$ Sunnybrook Health Sciences Centre, Toronto, Ontario, Canada

${ }^{9}$ Memorial Sloan Kettering Cancer Center, New York, Ontario, USA

${ }^{10}$ Dalla Lana School of Public Health, University of Toronto, Toronto, Ontario, Canada

${ }^{11}$ Applied Health Research Centre, Li Ka Shing Institute, St. Michael's Hospital,

Toronto, Ontario, Canada

Correction notice This article has been corrected since it was published. The caption of Figure 1 has been corrected. 
Contributors YB leads the study and conceived and designed the protocol. KET and $A L$ codesigned the protocol. MC codesigned the study and wrote the protocol. EG, KAS, MR, KO, JGH, JLE, AL, JCC and ME assisted in designing the decision aid. MC, SS, CM, EJ, SC, AS, CE, SP, AE, TG, MA, KMS and LWP assisted in data collection. SS, CM, EJ and SC assisted with drafting the manuscript. All authors read and approved the final manuscript.

Funding This study was supported by a foundation grant from the Canadian Institute of Health Research and a McLaughlin Centre Accelerator grant in genomic medicine (grant numbers 333703 and MC-2016-04, respectively). JGH, KO and MR were supported by NCI P30 CA008748.

Competing interests None declared.

Patient consent for publication Detail has been removed from this case description/these case descriptions to ensure anonymity. The editors and reviewers have seen the detailed information available and are satisfied that the information backs up the case the authors are making.

Ethics approval St. Michael's Hospital ethics board, Mount Sinai Hospital ethics board and Sunnybrook Health Sciences Centre ethics board.

Provenance and peer review Not commissioned; peer reviewed for ethical and funding approval prior to submission.

Open access This is an open access article distributed in accordance with the Creative Commons Attribution Non Commercial (CC BY-NC 4.0) license, which permits others to distribute, remix, adapt, build upon this work non-commercially, and license their derivative works on different terms, provided the original work is properly cited and the use is non-commercial. See: http://creativecommons.org/ licenses/by-nc/4.0/

(C) Article author(s) (or their employer(s) unless otherwise stated in the text of the article) 2018. All rights reserved. No commercial use is permitted unless otherwise expressly granted.

\section{REFERENCES}

1. Yang Y, Muzny DM, Xia F, et al. Molecular findings among patients referred for clinical whole-exome sequencing. JAMA 2014;312:1870-9.

2. Vissers L, van Nimwegen KJM, Schieving JH, et al. A clinical utility study of exome sequencing versus conventional genetic testing in pediatric neurology. Genet Med 2017;19:1055-63.

3. Parsons DW, Roy A, Yang Y, et al. Diagnostic yield of clinical tumor and germline whole-exome sequencing for children with solid tumors. JAMA Oncol 2016;2:616-24.

4. Genomics England. The 100,000 Genomes Project [Internet], 2017. https://www.genomicsengland.co.uk/the-100000-genomes-project/. (accessed 01 Dec 2017)

5. Mackley MP, Capps B. Expect the unexpected: screening for secondary findings in clinical genomics research. Br Med Bull 2017;122:109-22.

6. Clinical Genome Resource. Clinical Actionability [Internet]. 2018. https://www.clinicalgenome.org/curation-activities/clinicalactionability/results/ (accessed 01 Dec 2017).

7. Kalia SS, Adelman K, Bale SJ, et al. Recommendations for reporting of secondary findings in clinical exome and genome sequencing, 2016 update (ACMG SF v2.0): a policy statement of the American College of Medical Genetics and Genomics. Genet Med 2017;19:249-55.

8. Schwartz PJ, Crotti L, Insolia R. Long-QT Syndrome: From Genetics to Management. Circulation 2012;5:868-77.

9. Ploug T, Holm S. Clinical genome sequencing and population preferences for information about 'incidental' findings-From medically actionable genes (MAGs) to patient actionable genes (PAGs). PLoS One 2017;12:e0179935-13.

10. Vassy JL, Christensen KD, Schonman EF, et al. The Impact of Whole-Genome Sequencing on the Primary Care and Outcomes of Healthy Adult Patients: A Pilot Randomized Trial. Ann Intern Med 2017;167:159-69.

11. Bick D, Dimmock D. Whole exome and whole genome sequencing. Curr Opin Pediatr 2011;23:594-600.

12. Tabor HK, Stock J, Brazg T, et al. Informed consent for whole genome sequencing: a qualitative analysis of participant expectations and perceptions of risks, benefits, and harms. Am J Med Genet A 2012;158A:1310-9.

13. Stacey D, Bennett CL, Barry MJ, et al. Decision aids for people facing health treatment or screening decisions. Cochrane Database Syst Rev 2011:CD001431.
14. Bekker HL, Hewison J, Thornton JG. Applying decision analysis to facilitate informed decision making about prenatal diagnosis for Down syndrome: a randomised controlled trial. Prenat Diagn 2004;24:265-75.

15. Green MJ, Biesecker BB, Mclnerney AM, et al. An interactive computer program can effectively educate patients about genetic testing for breast cancer susceptibility. Am J Med Genet 2001;103:16-23.

16. Metcalfe KA, Dennis CL, Poll A, et al. Effect of decision aid for breast cancer prevention on decisional conflict in women with a BRCA1 or BRCA2 mutation: a multisite, randomized, controlled trial. Genet Med 2017;19:330-6.

17. Birch $\mathrm{PH}$. Interactive e-counselling for genetics pre-test decisions: where are we now? Clin Genet 2015;87:209-17.

18. Birch $\mathrm{P}$, Adam S, Bansback N, et al. Decide: a decision support tool to facilitate parents' choices regarding genome-wide sequencing. J Genet Couns 2016;25:1298-308.

19. Green RC, Berg JS, Grody WW, et al. ACMG recommendations for reporting of incidental findings in clinical exome and genome sequencing. Genet Med 2013;15:565-74.

20. Jarvik GP, Amendola LM, Berg JS, et al. Return of genomic results to research participants: the floor, the ceiling, and the choices in between. Am J Hum Genet 2014;94:818-26.

21. Khoury MJ, McBride CM, Schully SD, et al. The Scientific Foundation for personal genomics: recommendations from a National Institutes of Health-Centers for Disease Control and Prevention multidisciplinary workshop. Genet Med 2009;11:559-67.

22. O'Connor A. Ottawa Decision Support Framework to address decisional conflict: Ottawa Health Research Institute, 2006.

23. Bombard Y, Clausen M, Mighton C, et al. The Genomics ADvISER: Development and testing of a decision aid for the selection of incidental sequencing results. Eur J Hum Genet (In press).

24. Bredenoord AL, Kroes HY, Cuppen E, et al. Disclosure of individual genetic data to research participants: the debate reconsidered. Trends Genet 2011;27:41-7.

25. McGuire AL, Lupski JR. Personal genome research : what should the participant be told? Trends Genet 2010;26:199-201.

26. Bombard $\mathrm{Y}$, Clausen M, Glogowski E, et al.Genomics ADvISER: A Genomics Decision AiD about Incidental SEquencing Results. American Society of Human Genetics. Vancouver, BC, 2016.

27. Miller FA, Hayeems RZ, Bytautas JP, et al. Testing personalized medicine: patient and physician expectations of next-generation genomic sequencing in late-stage cancer care. Eur J Hum Genet 2014;22:391-5

28. Strauss A, Corbin J. Basics of qualitative research: techniques and procedures for developing grounded theory. 2nd Edition: Thousand Oaks, CA: Sage Publications, 1998.

29. Morse JM. Designing funded qualitative research. In: Denzin NKL, Lincoln YS, eds. Handbook of qualitative research. Thousand Oaks: Sage, 1994:220-35.

30. Sandelowski M. Sample size in qualitative research. Res Nurs Health 1995;18:179-83.

31. Coyne IT. Sampling in qualitative research. Purposeful and theoretical sampling; merging or clear boundaries? J Adv Nurs 1997;26:623-30.

32. Miles M, Huberman AH. Qualitative data analysis; an expanded sourcebook. Newbury Park, CA: Sage, 1994.

33. O'Connor AM. Validation of a decisional conflict scale. Medical Decision Making 1995;15:25-30.

34. Kaphingst KA, Facio FM, Cheng M-R, et al. Effects of informed consent for individual genome sequencing on relevant knowledge. Clin Genet 2012;82:408-15.

35. Holmes-Rovner M, Kroll J, Schmitt N, et al. Patient satisfaction with health care decisions: the satisfaction with decision scale. Med Decis Making 1996;16:58-64.

36. Bennett C, Graham ID, Kristjansson E, et al. Validation of a preparation for decision making scale. Patient Educ Couns 2010;78:130-3

37. Spielberger CD, Gorsuch RL, Lushene RE. The State-Trait Anxiety Inventory Test Manual. Palo Alto, CA, Consulting Psychologists Press 1970.

38. Julian LJ. Measures of Anxiety. Arthritis Care Res 2011;63:1-11.

39. Johnson K, Kieran S, Riordan S, et al. Genomic Counseling Guide: National Society of Genetic Counselors, 2014.

40. Laupacis A, O'Connor AM, Drake ER, et al. A decision aid for autologous pre-donation in cardiac surgery - a randomized trial. Patient Educ Couns 2006;61:458-66.

41. Wakefield CE, Meiser B, Homewood J, et al. Randomized trial of a decision aid for individuals considering genetic testing for hereditary nonpolyposis colorectal cancer risk. Cancer 2008;113:956-65.

42. Strauss A, Corbin J. Basics of Qualitative Research:Techniques and Procedures for Developing Grounded Theory. 2nd edn. Thousand Oaks, CA: Sage Publications, 1998. 
43. Strauss AL, Corbin J. Basics of qualitative research: grounded theory procedures and techniques. Newbury Park, CA: Sage, 1990.

44. Charmaz KC. Qualitative interviewing and grounded theory analysis. In: Holstein JA, Gubrium JF, eds. Inside interviewing: New lenses, new concerns. Thousand Oaks, CA: Sage Publications Inc, 2003:311-30.

45. Charmaz KC. Grounded theory: Objectivist and constructivist methods. In: Denzin NK, Lincoln YS, edseds. Handbook of
Qualitative Research. 2nd edn. Thousand Oaks, CA: Sage, 2000:509-35.

46. Charmaz KC. Constructing grounded theory: a practical guide through qualitative analysis. London: SAGE Publications Ltd, 2006.

47. Morse JM. Approaches to qualitative-quantitative methodological triangulation. Nurs Res 1991;40:120-3.

48. Sandelowski M. Rigor or rigor mortis: the problem of rigor in qualitative research revisited. ANS Adv Nurs Sci 1993;16:1-8. 